\title{
Genital schistosomiasis mansoni concomitant to genital tumor in areas of low endemicity: challenging diagnosis
}

\section{Authors}

Marta G Cavalcanti ${ }^{1}$

Margareth MI Gonçalves²

Magali M Barreto ${ }^{3}$

Aline Helen da Silva ${ }^{4}$

Kalil Madi ${ }^{4}$

José Mauro Peralta²,

Ricardo P Igreja ${ }^{5}$

${ }^{1}$ Infectious and Parasitic Diseases Service, Hospital Clementino Fraga Filho, Universidade Federal do Rio de Janeiro - HUCFF/ UFRJ, Brazil

${ }^{2}$ Instituto de Microbiologia Professor Paulo de Góes, UFRJ

${ }^{3}$ Department of Biology, Instituto Oswaldo Cruz, FIOCRUZ, Brazil

${ }^{4}$ Pathological Anatomy Service, HUCFF/UFRJ ${ }^{5}$ Department of Preventive Medicine, School of Medicine, UFRJ, Brazil

Submitted on: 02/10/2009 Approved on: 05/22/2009

Correspondence to: Marta G Cavalcanti Serviço de Doenças Infecciosas e Parasitárias Hospital Universitário Clementino Fraga Filho Universidade Federal do Rio de Janeiro

Rua Prof. Rodolfo Rocco $255-5^{\circ}$ andar Cidade Universitária Campus do Fundão 21 941-913, Rio de Janeiro, Brazil

Phone: 55-21-2562-2526 55-21-9156-8630 Fax: 55-21 25517023 cavalcanti@openlink. com.br

We declare no conflict of interest.

\begin{abstract}
Genital infection by Schistosoma mansoni is usually misdiagnosed in individuals who reside in, or travel to endemic areas. We describe two cases of genital tumor associated with $S$. mansoni infection manifested by methrorragy. Surgical specimens revealed leiomyomas in both cases associated with $S$. mansoni. In one of them, granulomas were found in the ovary and in the other they were found in the uterine tube. Although none presented intestinal/hepatic disease, fecal egg excretion was detected in one. Both had elevated pretreatment antibody reactivity to $S$. mansoni antigen, but follow-up showed different outcomes. Schistosomiasis should be considered as a diagnosis in individuals with methrorragy residing in or having traveled to endemic areas. Since diagnosis follows genital amputation, and cure control is troublesome, improvement of diagnostic tools and follow-up markers are important priorities to decrease schistosomiasis morbidity.
\end{abstract}

Keywords: S. mansoni; female genital infection; serology; low endemic area.

[Braz J Infect Dis 2011;15(2):174-177]@Elsevier Editora Ltda.

\section{INTRODUCTION}

Schistosomiasis is a chronic parasitic infection endemic in several countries in the tropics, which contributes to increased morbidity, and even mortality. Concern has grown, since schistosomiasis may progress to chronicity in the absence of any signs and symptoms of disease in individuals who are repeatedly or occasionally exposed to contaminated water, for example, travelers. ${ }^{1,2}$ Chronic infection is commonly characterized by intestinal and/ or hepatic involvement. However, reports of ectopic presentations even in individuals who are not residents of endemic areas and who travel or migrate from transmission areas of schistosomiasis are becoming more common. ${ }^{3,4}$ Ectopic presentations affect individuals with any parasitic load even after years of primary exposure. Among the ectopic presentations is genital schistosomiasis. Although Schistosoma hematobium is very often detected in the genital tract, other species may also be involved in genital infection, such as S. mansoni. . $^{5-7}$

Genital infection by $S$. mansoni is a lessrecognized complication of schistosomiasis even in areas of high endemicity. Prevalence of male and female genital schistosomiasis (FGS) is still unknown in South America. ${ }^{8}$ In FGS, clinical manifestations are often absent or resemble those of myomatosis and other genital affections, being frequently misdiagnosed. ${ }^{6,8}$ Usually, FGS is a postsurgical diagnosis. Also, no clinical or laboratory assays are shown to be reliable diagnostic or prognostic markers of infection or disease, and treatment efficacy is rarely assessed.

We report two FGS cases in individuals residing in Rio de Janeiro, Brazil, who presented methorragy and abdominal pain without a previous history of schistosomiasis. Postsurgical histopathology revealed eggs and granulomas in genital organs but only in one case eggs of $S$. mansoni in stool samples were found. Serum reactivity to $S$. mansoni adult worm antigen was detected before treatment with antiparasitic drugs in both patients. However, post-treatment serum antibody reactivity was distinct in each individual. The role of serological tests as a clinical marker of infection and/or disease has yet to be determined in these patients. 


\section{CASE REPORTS}

\section{Case 1}

Female, 42-years-old, born and raised in Paracambi (Rio de Janeiro, Brazil), during summer vacations used to go to Juiz de Fora (Minas Gerais, Brazil). Mother of two, had no history of abortions. She presented early symptoms of methrorragy, and abdominal pain four years before surgical intervention in 2004 . Histopathological analysis showed few granulomatous reactions and calcified eggs of $S$. mansoni in the left ovary (Figures $1 \mathrm{~A}$ and $\mathrm{B})$. Examination of the uterus also demonstrated presence of adenomyosis and leiomyomas. Abdominal ultrasound did not show signs of hepatosplenic or portal disturbances. No eggs were detected in the stool samples. Pretreatment serum levels of IgG1 and IgE anti-S. mansoni adult worm determined by in-house ELISA ${ }^{9}$ were elevated, as shown in Table 1. Praziquantel at a dose of $40 \mathrm{mg} / \mathrm{kg}$ was prescribed, and two years thereafter the levels of antibody isotypes were undetectable.

\section{Case 2}

Female, 40-years-old, born in Minas Gerais, living in Rio de Janeiro (Brazil) for the past 24 years, presented with intense methrorragy and pelvic pain for two years before admission in January, 2006 at the HUCFF, Universidade Federal do Rio de Janeiro. The patient reported urinary incontinency in the past four years and an ovarian cyst three years earlier. Mother of three, she had an abortion in 1988. An USG showed uterine intramural nodules in the posterior and anterior wall, respectively, and cysts were also detected in the right ovary and on the left annex. The patient had a history of hypertension, type II diabetes, and dyslipidemia since January, 2006. In June, 2006, subtotal hysterectomy and excision of both right and left ovaries were performed, and histopathological analysis showed Schistosoma mansoni eggs, several granulomas at the left uterine tube, and uterine leiomyoma (Figures $1 \mathrm{C}$ and D).

Figure 1: Schistosoma mansoni eggs and granulomatous reaction in female genital organs. In (A) and (B), histopathological examination of surgical specimen from patient 1 revealed two non-viable eggs surrounded by intense granulomatous reaction in one of the ovaries (hematoxylineosin, original magnification $\times 200$ and $\times 400$, respectively). A section of the left uterine tube from patient 2 showed intense granulomatous reaction, extensive fibrosis, and the presence of numerous viable parasitic ova as demonstrated by H\&E in (C), and Masson trichrome and Gomori stain in (D) (magnification $\times 200$ and $\times 400$, respectively). White arrows indicate viable $S$. mansoni egg and black arrow indicates calcified egg. Asterisk represents granulomatous reaction.

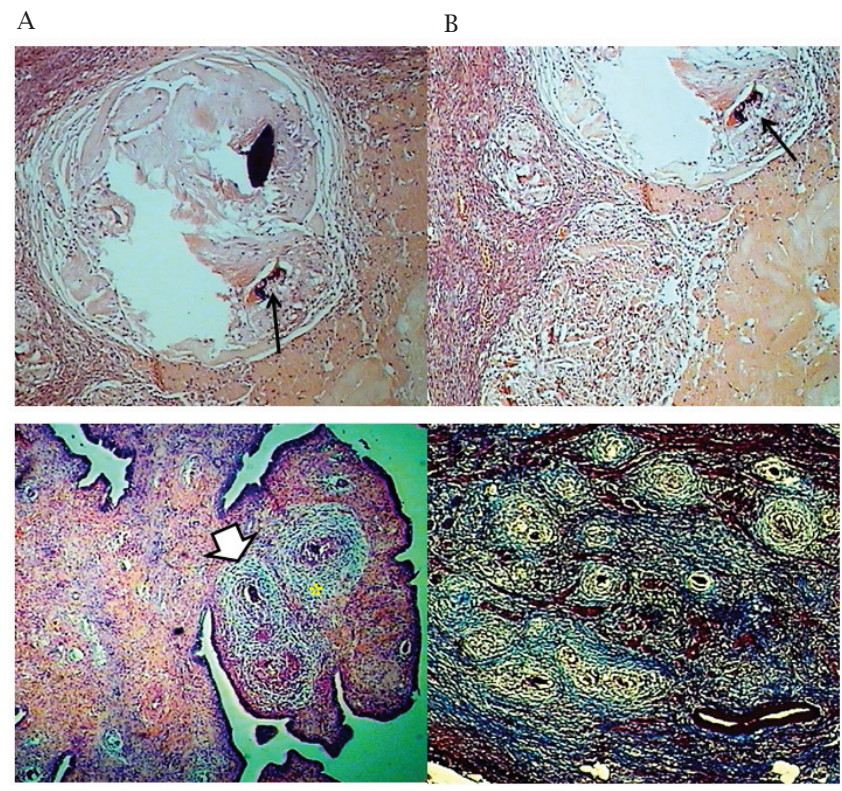

C

D

Table 1. Pre- and post-treatment parasitological and serological results of patients with genital infection by S. mansoni

\begin{tabular}{|c|c|c|c|c|c|c|c|c|}
\hline \multirow[t]{2}{*}{ Patients } & & \multirow[t]{2}{*}{ Histopathology } & \multirow[t]{2}{*}{$\begin{array}{l}\text { Coproscopy } \\
\text { eggs/ga }\end{array}$} & \multirow[t]{2}{*}{$\begin{array}{l}\text { Rectal } \\
\text { biopsy }\end{array}$} & \multicolumn{4}{|c|}{$\begin{array}{l}\text { Antibody titer } \\
\text { (ELISA units) }\end{array}$} \\
\hline & & & & & IgG & IgG1 & IgG4 & IgE \\
\hline \multicolumn{9}{|l|}{$\mathrm{P} 1$} \\
\hline & Pre- Rx & Calcified eggs & Negative & $\mathrm{ND}^{\mathrm{c}}$ & 0.90 & 2.10 & 0.40 & 1.10 \\
\hline & Post- Rx & & ND & & 0.64 & 0.38 & 0.25 & 0.40 \\
\hline \multicolumn{9}{|l|}{$\mathrm{P} 2$} \\
\hline & Pre- $\mathrm{Rx}$ & Viable eggs & & & 3.80 & 3.20 & 4.00 & 3.40 \\
\hline & Post $1^{\text {st }} \mathrm{Rx}$ & & & & & & & \\
\hline & $6 \mathrm{mo}$ & & Negative & & 3.94 & 3.14 & 3.90 & 3.52 \\
\hline & $12 \mathrm{mo}$ & & & & 2.54 & 2.39 & 0.90 & 4.85 \\
\hline & $20 \mathrm{mo}$ & & Negative & Viable eggs & 2.77 & 2.92 & 1.80 & 2.81 \\
\hline
\end{tabular}

a Number of eggs per gram of feces determined by Kato-Katz method

${ }^{\mathrm{b}}$ ELISA units determined as DO from serum sample/DO from the cut-off of the test

${ }^{\mathrm{c}} \mathrm{ND}$ - not determined

Rx- treatment with antiparasitic drug. 
In September, the patient was admitted to the Infectious Diseases Clinic. Clinical history and examination revealed no signs and symptoms of enteric or hepatosplenic disease. No portal hypertension was detected by ultrasound, which revealed slight hepatic steatosis compatible with the diagnosis of obesity (grade III). Coproscopy detected 696 eggs /g of feces and IgG, IgG1, IgG4, and IgG E serum levels were increased in two different samples before therapy (Table 1). At the time, only oxamniquine was available and administered at a dose of $15 \mathrm{mg} / \mathrm{kg}$, but a strong gastrointestinal reaction ensued. Despite subsequent post-treatment stool samples being negative, IgG1 and IgG4 levels were particularly elevated for 6, 16, and 20 months after treatment, as shown in Table 1. Rectal biopsy was performed and demonstrated the presence of viable eggs of $S$.mansoni. Praziquantel $(40 \mathrm{mg} / \mathrm{kg}$ of body weight) was indicated.

\section{DISCUSSION}

Schistosomiasis is a chronic infection which is one of the leading causes of high morbidity in tropical areas. It affects not only residents of endemic areas, but also immigrants and travelers who are no longer exposed to S. mansoni infection. ${ }^{2,10}$ Usually infected individuals present as asymptomatic. However, intestinal and/or hepatosplenic disease are current abnormalities in individuals from areas with high or even low endemicity. ${ }^{11}$ Like other Schistosoma species, S. mansoni may also induce pathology in other sites such as the lower and upper genital tract. ${ }^{8}$

Upper genital infection by Schistosoma spp. in women is frequently disguised as tumors or other genital diseases. Differential diagnosis is challenging in women presenting methrorragy as the main clinical finding. ${ }^{8,12}$ The present report describes two cases of female genital infection in patients residing in different geographical areas of Rio de Janeiro State, Brazil. The two patients showed clinical findings which could not be distinguished from benign or malignant genital diseases. In one of the cases, presurgical USG showed cysts in the right ovary and left annex, besides uterine mural nodules. In both cases, surgical specimen demonstrated the presence of cysts in ovaries and also in the annex in one patient. Both presented uterine leiomyoma. In S. haematobium infection, lower genital infection may present with characteristic lesions but upper infection lacks particular clinical presentation. ${ }^{6}$ Regarding $S$. mansoni infection, the manifestations of lower and upper genital tract infection remain elusive. Other concomitant conditions may also respond for the signs and symptoms of genital disease, which might overlap with S. mansoni infection manifestations. Association with other tumors and malignancies has been described in female genital infection by S. haematobium. ${ }^{7,12}$ However, its role in tumorigenesis is still speculative, and no evidence points to the involvement of $S$. mansoni in either of the cases.
In schistosomal upper-genital infection, diagnosis is usually based on histopathological examination of surgical specimens. ${ }^{6,7}$ However, detection of eggs in atypical sites does not rule out infection in traditional ones. Nonetheless, detection of ova excretion and serology are used less frequently as diagnostic tools in those cases. Long-term followup for cure control is rarely assessed. We found that despite the absence of clinical history of schistosomiasis, one of the patients presented eggs in the stools. Possible treatment failure also resulted in maintained egg excretion as seen in the rectal biopsy, and persistent reactivity in serological tests. In contrast, the first patient who presented only calcified eggs in the genital tissue, and no ova excretion had negative reactivity to schistosoma antigens post-treatment. This data suggest that detection of egg excretion and serology are important diagnostic tools to determine concomitant infection in other sites. Further studies are warranted to evaluate the role of combined parasitological and immunological tests as markers for treatment response in individuals with different presentations of schistosomal infection.

In conclusion, female genital infection by $S$. mansoni is a silent chronic infection that may occur even in individuals who no longer live in endemic areas. Concomitant infection of other sites must also be investigated through detection of ova excretion and serum reactivity to $S$. mansoni. Further, cure control might be determined by the combination of both parasitological and immunological assays.

\section{ACKNOWLEDGMENTS}

We thank Dr Helena Ribeiro LaGrega, Dra Vera Lucia Chagas (HUCFF/UFRJ, Rio de Janeiro, Brazil) and Dr Carlos Santos Pereira, Laboratório LUPA (Rio de Janeiro, Brazil) for their invaluable cooperation, and $\mathrm{CNPq}$ (Brazil) for financial support.

\section{REFERENCES}

1. Boers KE, Sastrowijoto PH, Elsakker van EP, and Hermans MP. Schistosomiasis of the uterus in a patient with dysmenorrheal and menorrhagia. Eur. J. Obstet. Gynecol. Reprod Biol., 2003; 108:106-108.

2. Raglio A, Russo V, Swierczynski G, Sonzogni A, Goglio A, Garcia LS. Acute Schistosomiasis mansoni infection with progression to chronic lesion in Italian travelers returning from Cameroon, West Africa: diagnostic and prevention problem. J Travel Med., 2002; 9:101-102.

3. Pérignon A, Pelicot M, Consigny PH. Genital Schistosomiasis in a traveler coming back from Mali. J. Travel Med., 2007; 14:197-199.

4. Sheorey H, Charles PGP, Pyman J. Ectopic Schistosomiasis in a returned traveler. J Travel Med., 2004; 11:251-252.

5. Hoffmann H, Bauerfeind I. High tissue egg burden mechanically impairing the tubal motility in genital schistosomiasis of the female. Acta. Obstect. Gynecol. Scand., 2003; 82:970-971

6. Ponggensee G, Feldmeier H, Krantz I. Schistosomiasis of the female genital tract: public health aspects. Parasitol Today. 1999; 15:378-381. 
7. Swai B, Ponggensee G, Mtweve S, Krantz I. Female genital schistosomiasis as an evidence of a neglected cause for reproductive ill-health: a retrospective histopathological study from Tanzania. BMC Infect. Dis., 2006; 6:134-141.

8. Oliveira FAS, Soares VL, Dacal RC et al. Absence of cervical schistosomiasis among women from two areas of north-eastern Brazil with endemic Schistosoma mansoni. Ann. Trop. Med. Parasitol. 2006; 100:49-54.

9. Gonçalves MM, Barreto MG, Peralta RH et al. Immunoassays as an auxiliary tool for the serodiagnosis of Schistosoma mansoni infection in individuals with low intensity of egg elimination. Acta Trop., 2006; 100:24-30.
10. Posey DL, Blackburn BG, Weinberg M et al. High prevalence and presumptive treatment of schistosomiasis and strongyloidiasis among African refugees. Clin Infect Dis., 2007; 45: 1310-1315.

11. Igreja RP, Matos JA, Gonçalves MM, Barreto MM, Peralta JM. Schistosoma mansoni-related morbidity in a low prevalence area in Brazil: a comparison between egg-excretors and $S$. mansoni antibody positive individuals. Ann Trop Med Parasitol. 2007; 101(7):575-584.

12. Helling-Giese G, Kjetland EF, Gundersen SG et al. Schistosomiasis in women: manifestations in the upper reproductive tract. Acta Trop., 1996; 62:225-238. 